\title{
UNIQUENESS, STRONG FORMS OF UNIQUENESS AND NEGATIVE POWERS OF CONTRACTIONS
}

\author{
JEAN ESTERLE \\ CeReMaB, Université Bordeaux I \\ 351, cours de la Libération, 33405 Talence, France
}

1. Introduction. The notion of uniqueness goes back to Cantor: a subset $E$ of the unit circle $\Gamma$ is said to be a set of uniqueness if the zero sequence is the only sequence $\left(c_{n}\right)_{n \in \mathbb{Z}}$ of complex numbers such that

$$
\sum_{|n| \leq m} c_{n} e^{i n t} \underset{m \rightarrow \infty}{\longrightarrow} 0
$$

for every $e^{i t} \notin E$. A set of multiplicity is a set which is not a set of uniqueness. Cantor [5] showed in 1870 that finite sets (in particular the empty set) are sets of uniqueness, and more generally that "reducible" countable sets are sets of uniqueness. It was only in 1908 that Young [32] was able to prove that all countable sets are sets of uniqueness.

We will be only interested here in closed sets (we refer to Kechris-Louveau [20] for a discussion of the notion of uniqueness for nonclosed sets). In this case $E$ is a set of multiplicity if and only if there exists a nonzero pseudofunction, i.e. a distribution $S$ (see Section 2) such that

$$
\widehat{S}(n) \underset{|n| \rightarrow \infty}{\longrightarrow} 0,
$$

with support contained in $E$. In other terms, if we denote by $P F(E)$ the set of pseudofunctions $S$ such that Supp $S \subset E$, then $E$ is a set of uniqueness if and only if $\operatorname{PF}(E)=\{0\}$. It follows immediately from this fact that if $E$ has positive Lebesgue measure then $E$ is a set of multiplicity.

In Section 2 we describe some classical results concerning uniqueness, including the famous Salem-Zygmund characterization of perfect symmetric sets of constant ratio which are sets of uniqueness [28].

1991 Mathematics Subject Classification: 42A20, 46J20, 47D03.

The paper is in final form and no version of it will be published elsewhere. 
An obvious strengthening of the notion of uniqueness is obtained by considering the set $P M^{0}(E)$ of all pseudomeasures $S$ (i.e. distributions $S$ such that $\left.\sup _{n \in \mathbb{Z}}|\widehat{S}(n)|<\infty\right)$ such that $\operatorname{Supp} S \subset E$ and $\lim _{n \rightarrow-\infty} \widehat{S}(n)=0$. Clearly, $P F(E) \subset P M^{0}(E)$ and so any closed set $E$ such that $P M^{0}(E)=\{0\}$ is a set of uniqueness. It follows from the results of Kahane-Katznelson [16] that "strong $A A^{+}$-sets" (see Section 2) do possess this strong form of uniqueness.

Now denote by $H D(\Gamma)$ the set of all hyperdistributions on $\Gamma$ (see Section 2) and set $H D^{0}(\Gamma)=\left\{S \in H D(\Gamma) \mid \lim _{n \rightarrow-\infty} \widehat{S}(n)=0\right\}$; if $E \subset \Gamma$ is closed, set $H D^{0}(E)=\left\{S \in H D^{0}(\Gamma) \mid \operatorname{Supp} S \subset E\right\}$.

Let $\omega: \mathbb{Z}^{+} \rightarrow[1, \infty[$ be a submultiplicative weight. Let

$$
P M_{\omega}^{0}(E)=\left\{S \in H D^{0}(E)|\sup | \widehat{S}(n) \mid / \omega(n)<\infty\right\} .
$$

We will say that $E$ is a set of $\omega$-uniqueness if $P M_{\omega}^{0}(E)=\{0\}$. Since hyperdistributions can be interpreted as analytic functions on $\mathbb{C} \backslash \Gamma$ vanishing at infinity there are obvious restrictions to these strong forms of uniqueness related to inner functions: For every nonempty closed set $E$ and every $\varepsilon>0$ there exists a nonzero $S \in H D^{0}(E)$ such that

$$
\limsup _{n \rightarrow \infty} \frac{\log ^{+}|\widehat{S}(n)|}{\sqrt{n}}<\varepsilon,
$$

and for every uncountable closed set $E$ there exists a nonzero $S \in H D^{0}(E)$ such that $\lim _{n \rightarrow \infty}\left(\log ^{+}|\widehat{S}(n)|\right) / \sqrt{n}=0$. On the other hand, if $S \in H D^{0}(\Gamma)$ satisfies

$$
\frac{\log ^{+}|\widehat{S}(n)|}{\sqrt{n}} \underset{n \rightarrow \infty}{\longrightarrow} 0
$$

then Supp $S$ is a perfect set, and so countable sets have the $\omega$-uniqueness property for all weights $\omega$ such that $\lim _{n \rightarrow \infty}(\log \omega(n)) / \sqrt{n}=0$ (see Zarrabi [34]). We present a new proof of this result, based on analytic methods, in Section 3.

Now denote by $E_{1 / p}$ the perfect symmetric set of constant ratio $1 / p$. We show in Section 4 that every distribution $S$ supported by $E_{1 / p}$ such that $\lim _{n \rightarrow-\infty} \widehat{S}(n)$ $=0$ vanishes. Stronger results are known, but the class of weights $\omega$ for which $E_{1 / p}$ has the $\omega$-uniqueness property remains to be determined.

In Section 5 we show that no general $\omega$-uniqueness property holds for Dirichlet sets: given any weight $\omega$ such that $\lim _{n \rightarrow \infty} \omega(n)=\infty$ there exists a Dirichlet set $E$ and a nonzero distribution $S \in P M_{\omega}^{0}(E)$. A similar result holds for Kronecker sets [9], but the proof for Dirichlet sets, which is significantly simpler, is probably more suitable to understand the nature of the phenomenon (which is somewhat related to Kaufman's construction [19] of Helson sets of multiplicity).

In Section 6 we discuss a related, stronger property [9]. A closed set $E \subset \Gamma$ is said to be $\omega$-rigid if $P M_{\omega}(E)=P M(E)$ where $P M_{\omega}(E)$ is the set of all hyperdistributions $S$ with support contained in $E$ which satisfy

$$
\sup _{n<0}|\widehat{S}(n)|<\infty, \quad \sup _{n \geq 0} \frac{|\widehat{S}(n)|}{\omega(n)}<\infty .
$$


If $E$ is $\omega$-rigid for some unbounded weight $\omega$, then $E$ is a "strong" $A A^{+}$-set, and for strong $A A^{+}$-sets $\omega$-rigidity is equivalent to $\omega$-uniqueness. This notion has applications to operator theory: when $E$ is $\omega$-rigid, every contraction $T$ on a Banach space such that $\operatorname{Sp} T \subset E$ and $\sup _{n \geq 1}\left\|T^{-n}\right\| / \omega(n)<\infty$ satisfies in fact $\sup _{n \geq 0}\left\|T^{-n}\right\|<\infty$. Conversely, if the above property holds for every Banach space $X$, then $E$ is $\omega$-rigid. Examples given in Section 6 show that this converse does not hold if we restrict attention to Hilbert spaces.

To conclude this review we indicate in Section 7 the link between these strong forms of uniqueness and the structure of closed ideals of $A^{+}$, the algebra of absolutely convergent Taylor series. The results of Section 4 imply that a closed ideal $I$ of $A^{+}$such that $h(I) \cap \Gamma=E_{1 / p}$, where $h(I)$ is the hull of $I$, is entirely determined by its inner factor [10]. This proves, in a special case, an old conjecture of Bennett and Gilbert [3]. On the other hand, it follows from the results of Section 5 that this conjecture is not true in general [9].

\section{Classical uniqueness theory}

Definition 2.1 [17]. A hyperdistribution is a holomorphic function $S$ on $\mathbb{C} \backslash \Gamma$ such that $S(z) \rightarrow 0$ as $|z| \rightarrow \infty$. The Fourier coefficients of $S$ are defined by the formulae

$$
\begin{aligned}
S(z) & =\sum_{n \geq 1} \widehat{S}(n) z^{n-1} \quad(|z|<1), \\
S(z) & =-\sum_{n \leq 0} \widehat{S}(n) z^{n-1} \quad(|z|>1) .
\end{aligned}
$$

A point $z_{0} \in \Gamma$ is said to be regular for $S$ if there exist $\varrho>0$ and $F_{z_{0}}$ holomorphic on $D\left(z_{0}, \varrho\right)$ such that $S(z)=F_{z_{0}}(z)$ for $z \in D\left(z_{0}, \varrho\right),|z| \neq 1$. Nonregular points are said to be singular, and the support of $S$, denoted by Supp $S$, is the set of singular points of $S$.

A hyperdistribution $S$ is said to be $\left({ }^{1}\right)$

- a pseudofunction if $\widehat{S}(n) \rightarrow 0$ as $|n| \rightarrow \infty$;

- a pseudomeasure if $\|S\|_{P M}=\sup _{n \in \mathbb{Z}}|\widehat{S}(n)|<\infty$;

- a distribution if $|\widehat{S}(n)|=O\left(|n|^{k}\right)$ as $|n| \rightarrow \infty$ for some $k \geq 0$.

We denote by $P M(\Gamma), P F(\Gamma), D(\Gamma), H D(\Gamma)$ the sets of all pseudomeasures, pseudofunctions, distributions, and hyperdistributions. Also if $E \subset \Gamma$ we denote by $P F(E)$ the set of all pseudofunctions $S$ such that Supp $S \subset E$, and we define in the same way $P M(E), D(E)$, and $H D(E)$. It follows immediately from Liouville's theorem that Supp $S \neq \emptyset$ for every nonzero $S \in H D(\Gamma)$.

We summarize the link between uniqueness and pseudofunctions in the following theorem [17, Chapter 5, §4].

$\left({ }^{1}\right)$ Editorial note: See also the theorems of G. Pólya and S. M. Shah cited on pp. 371-372 in this volume. 
TheOREM 2.2. Let $E \subsetneq \Gamma$ be a closed set, and let $\left(c_{n}\right)_{n \in \mathbb{Z}}$ be a family of complex numbers. We have:

1) If $\lim _{m \rightarrow \infty} \sum_{|n| \leq m} c_{n} e^{i n t}=0$ for $e^{i t} \in \Gamma \backslash E$, then $c_{n} \rightarrow 0$ as $|n| \rightarrow \infty$.

2) If $c_{n} \rightarrow 0$ as $|n| \rightarrow \infty$, set

$$
\begin{array}{ll}
S(z)=\sum_{n=0}^{\infty} c_{n} z^{n} & (|z|<1), \\
S(z)=-\sum_{n=-\infty}^{-1} c_{n} z^{n} & (|z|>1) ;
\end{array}
$$

then the following conditions are equivalent:

(i) $\lim _{m \rightarrow \infty} \sum_{|n| \leq m} c_{n} e^{i n t}=0$ for every $e^{i t} \in \Gamma \backslash E$;

(ii) $\operatorname{Supp} S \subset E$.

We thus see that $E$ is a set of uniqueness if and only if $P F(E)=\{0\}$. The notion of uniqueness can also be interpreted in terms of the classical Wiener algebra

$$
A(\Gamma)=\left\{f \in \mathcal{C}(\Gamma)\left|\|f\|_{1}=\sum_{n \in \mathbb{Z}}\right| \widehat{f}(n) \mid<\infty\right\},
$$

the algebra of absolutely convergent Fourier series; $\left(A(\Gamma),\|\cdot\|_{1}\right)$ is a regular Banach algebra, and the characters of $A(\Gamma)$ are the morphisms $\chi_{z}: f \rightarrow f(z)$ where $z \in \Gamma$.

Let $I(E)=\left\{\left.f \in A(\Gamma)|| f\right|_{E}=0\right\}$ and denote by $J(E)$ the closure in $(A(\Gamma)$, $\left.\|\cdot\|_{1}\right)$ of the set of all $f \in A(\Gamma)$ such that Supp $f \cap E=\emptyset$, where we denote by Supp $f$ the closed support of $f$.

If $I$ is a closed ideal of $A(\Gamma)$ set $h(I)=\{z \in \Gamma \mid f(z)=0(f \in I)\}$. Then it follows from standard results about regular algebras [18] that

$$
h(I(E))=h(J(E))=E \quad \text { and } \quad J(E) \subset I \subset I(E)
$$

for each closed ideal $I$ of $A(\Gamma)$ such that $h(I)=E$. We can identify $A(\Gamma)$ with the dual of $P F(\Gamma)$ and $P M(\Gamma)$ with the dual of $A(\Gamma)$ by using the formula

$$
\langle f, S\rangle=\sum_{n \in \mathbb{Z}} \widehat{f}(-n) \widehat{S}(n) \quad(f \in A(\Gamma), S \in P M(\Gamma)),
$$

and routine computations show that we have

$$
\langle f, S\rangle=\lim _{r \rightarrow 1^{-}} \frac{1}{2 i \pi} \int_{\Gamma} f(\zeta)[S(r \zeta)-S(\zeta / r)] d \zeta .
$$

It follows from (2.4) that $\langle f, S\rangle=0$ if $\operatorname{Supp} f \cap \operatorname{Supp} S=\emptyset$, so that $P M(E) \subset$ $[J(E)]^{\perp}$.

To obtain the converse, denote by $\alpha: z \rightarrow z$ the identity map on $\Gamma$. Then $\widehat{\alpha^{p}}(p)=1$ and $\widehat{\alpha^{p}}(n)=0$ for $n \neq p$, so that $\widehat{S}(n)=\left\langle\alpha^{-n}, S\right\rangle$ for $n \in \mathbb{Z}$. 
So we have the formula

$$
\left\langle(\alpha-z)^{-1}, S\right\rangle=S(z) \quad(z \in \mathbb{C} \backslash \Gamma, S \in P M(\Gamma)) .
$$

Now assume that $S \in J(E)^{\perp}$ and let $\pi: A(\Gamma) \rightarrow A(\Gamma) / J(E)$ be the canonical map. Since $h(J(E))=E$, characters of $A(\Gamma) / J(E)$ have the form $\pi(f) \rightarrow f(z)$ for some $z \in E$, and $\operatorname{Sp} \pi(\alpha)=E$. For $f \in A(\Gamma)$ set $\langle\pi(f), L\rangle=\langle f, S\rangle$.

Then $L$ is a continuous linear functional on $A(\Gamma) / J(E)$. If we set $\widetilde{S}(z)=$ $\left\langle(\pi(\alpha)-z)^{-1}, L\right\rangle$, we obtain an analytic extension of $S$ to $\mathbb{C} \backslash E$, so that $\operatorname{Supp} S \subset E$ (the function $\widetilde{S}$ is the "Domar-Gelfand transform of $S$ " [8]; curiously enough, the fact that $J(E)^{\perp} \subset P M(E)$ is usually proved in a much more complicated way). Hence we have the following standard result.

Proposition 2.6. For every closed set $E \subset \Gamma, P M(E)=[J(E)]^{\perp}$.

The $w^{*}$-topology in $A(\Gamma)$ is, by definition, the weak topology $\sigma(A(\Gamma), P F(\Gamma))$ on $A(\Gamma), A(\Gamma)$ being identified with the dual space of $P F(\Gamma)$ (the $w^{*}$-topology agrees with the topology of pointwise convergence of Fourier coefficients on bounded subsets of $A(\Gamma))$.

It follows from Theorem 2.2 and Proposition 2.6 that we have the following characterization of sets of uniqueness:

Corollary 2.7. Let $E \subset \Gamma$ be a closed set. Then the following conditions are equivalent:

(1) $E$ is a set of uniqueness.

(2) $P F(E)=\{0\}$.

(3) $J(E)$ is $w^{*}$-dense in $A(\Gamma)$.

A function $f \in A(\Gamma)$ satisfies synthesis for $E$, by definition, iff $f \in J(E)$. If $J(E)=I(E)$, which means that all functions vanishing on $E$ satisfy synthesis for $E$, then $E$ is a set of synthesis. We thus see that a set of synthesis $E$ is a set of uniqueness if and only if $I(E)$ is $w^{*}$-dense in $A(\Gamma)$ (for a discussion of $w^{*}$-density see for example [11, Chapter 4]).

Now if $\mu$ is a regular Borel measure on $\Gamma$ (which we write as a periodic measure on $\mathbb{R})$ set

$$
S_{\mu}(z)=\frac{1}{2 \pi} \int_{0}^{2 \pi} \frac{d \mu(t)}{e^{i t}-z} \quad(z \in \mathbb{C} \backslash \Gamma) .
$$

Then $S_{\mu} \in P M(E)$, where $E$ is the closed support of $\mu$, and $\widehat{S}_{\mu}(n)=\widehat{\mu}(n)$ $(n \in \mathbb{Z})$. Identifying $\mu$ with $S_{\mu}$, we thus see that a measure is a pseudomeasure, and an $L^{1}$-function is a pseudofunction. In particular, every closed set of positive Lebesgue measure is a set of multiplicity, since its characteristic function is a nonzero pseudofunction. The map $\mu \rightarrow S_{\mu}$ extends in an obvious way to an isomorphism of the dual space of $\mathcal{C}^{\infty}(\Gamma)$ (distributions in the usual sense) into the set $D(\Gamma)$ introduced in Definition 2.1, and $\operatorname{Supp} \mu=\operatorname{Supp} S_{\mu}$. 
For $\zeta \in(0,1 / 2)$ denote by

$$
E_{\zeta}=\left\{\exp \left(2 i \pi \sum_{n \geq 1} \varepsilon_{n} \zeta^{n-1}(1-\zeta)\right) \mid \varepsilon_{n}=0 \text { or } 1\right\}
$$

the perfect symmetric set of constant ratio $\zeta$. Recall that $t>0$ is said to be a Pisot number if there exist integers $a_{0}, \ldots, a_{n-1}$ such that $t^{n}+a_{n-1} t^{n-1}+\ldots+a_{0}=0$ and such that all other roots of the above equation lie in the open unit disc.

The Salem-Zygmund theorem ([28] or [17, Chapter 6]) gives the following characterization of uniqueness for the sets $E_{\zeta}$.

THEOREM 2.8. $E_{\zeta}$ is a set of uniqueness if and only if $1 / \zeta$ is a Pisot number.

When $1 / \zeta$ is not a Pisot number, a nonzero element of $P F\left(E_{\zeta}\right)$ is given by a nonatomic measure concentrated on $E_{\zeta}$, but there are examples of sets of multiplicity $E$, due to Pyateckiı-Shapiro [25], for which $P F(E)$ does not contain any nonzero measure [11, p. 104].

Now denote by $A^{+}$the algebra of absolutely convergent Taylor series, i.e. the algebra of analytic functions $f$ on the open unit disc such that

$$
\|f\|_{1}=\sum_{n=0}^{\infty} \frac{\left|f^{(n)}(0)\right|}{n !}<\infty .
$$

By identifying elements of $A^{+}$with their restrictions to the unit circle, we can isometrically identify $A^{+}$with the algebra $\{f \in A(\Gamma) \mid \widehat{f}(n)=0(n<0)\}$. A set $E$ is said to be a set of $A A^{+}$-interpolation, or an $A A^{+}$-set, if $A^{+}(E)=A(E)$ where we denote by $A^{+}(E)$ (resp. $A(E)$ ) the algebra of restrictions to $E$ of elements of $A^{+}$(resp. $\left.A(\Gamma)\right)$. In other terms, $E$ is an $A A^{+}$-set if and only if $I(E)+A^{+}=$ $A(\Gamma)$. This suggests the following definition:

Definition 2.9. A closed set $E \subset \Gamma$ is a strong $A A^{+}$-set if $J(E)+A^{+}=$ $A(\Gamma)$. If $E$ is a strong $A A^{+}$-set, the strong $A A^{+}$-constant of $E$ is $\left\|i^{-1}\right\|$ where $i: f+J^{+}(E) \rightarrow f+J(E)$ is the natural injection from $A^{+} / J^{+}(E)$ into $A(\Gamma) / J(E)$.

By using duality arguments, Kahane and Katznelson showed in [16, Theorem 2.1] that $E$ is an $A A^{+}$-set if and only if there exists $K>0$ such that $\|S\|_{P M} \leq K \lim \sup _{n \rightarrow \infty}|\widehat{S}(-n)|$ for every pseudomeasure $S \in I(E)^{\perp}$, where $\|S\|_{P M}=\sup _{n \in \mathbb{Z}}|\widehat{S}(n)|$, and the $A A^{+}$-constant of $E$, defined as the norm of the inverse of the natural injection from $A^{+}(E)$ into $A(E)$, is the smallest $K$ satisfying the inequality above. Also it was noticed in [35] that $E$ is an $A A^{+}$-set if and only if $I^{+}(E)$ is $w^{*}$-sequentially dense in $A^{+}$(the $w^{*}$-topology on $A^{+}$is obtained by identifying $A^{+}$to the dual of $c_{0}$, and it is the restriction to $A^{+}$of the $w^{*}$-topology on $A(\Gamma))$. The following proposition extends these results to strong $A A^{+}$-sets.

Proposition 2.10. Let $E \subset \Gamma$ be a closed set. Then the following conditions imply each other:

(1) $E$ is a strong $A A^{+}$-set.

(2) $J^{+}(E)$ is $w^{*}$-sequentially dense in $A^{+}$. 
(3) There exists $K>0$ such that

$$
\|S\|_{P M} \leq K \limsup _{n \rightarrow-\infty}|\widehat{S}(n)| \quad(S \in P M(E)) .
$$

Moreover, the strong $A A^{+}$-constant of $E$ is the smallest $K>0$ satisfying (3).

Proof. Denote by $\pi: A(\Gamma) \rightarrow A(\Gamma) / J(E)$ and $\pi^{+}: A^{+} \rightarrow A^{+} / J^{+}(E)$ the canonical maps. If $E$ is a strong $A A^{+}$-set, let $K$ be the strong $A A^{+}$-constant of $E$.

Then $\left\|\pi^{+}(\alpha)^{-n}\right\| \leq K\left\|\pi(\alpha)^{-n}\right\|=K$ and so there exist $f_{n} \in A^{+}$and $\varphi_{n} \in$ $J^{+}(E)$ such that $\left\|f_{n}\right\| \leq K+1 / n$ and $\alpha^{n} f_{n}=1+\varphi_{n}$. Since $f_{n} \in A^{+}$, the sequence $\left(-\varphi_{n}\right)_{n \geq 1}$ converges $w^{*}$ to 1 and $J^{+}(E)$ is $w^{*}$-sequentially dense in $A^{+}$. Conversely, assume that there exists a sequence $\left(\psi_{n}\right)_{n \geq 1}$ in $J^{+}(E)$ which converges $w^{*}$ to 1 . It follows from the Banach-Steinhaus theorem that $M=$ $\limsup _{n \rightarrow \infty}\left\|\psi_{n}\right\|_{1}<\infty$, and $\lim _{n \rightarrow \infty} \widehat{\psi}_{n}(0)=1, \lim _{n \rightarrow \infty} \widehat{\psi}_{n}(m)=0$ for $m \geq 1$. Fix $p \geq 1$ and set

$$
U_{n, p}=\sum_{m=0}^{p-1} \widehat{\psi}_{n}(m) \alpha^{m}, \quad R_{n, p}=\sum_{m=p}^{\infty} \widehat{\psi}_{n}(m) \alpha^{m-p} .
$$

Then $\lim _{n \rightarrow \infty}\left\|1-U_{n, p}\right\|_{1}=0$, and so $U_{n, p}$ is invertible when $n$ is large enough, and $\lim _{n \rightarrow \infty}\left\|U_{n, p}^{-1}\right\|=1$. We obtain $1=\left(\psi_{n}-\alpha^{n} R_{n, p}\right) U_{n, p}^{-1}$ and so

$$
\left\|\pi^{+}(\alpha)^{-p}\right\| \leq \limsup _{n \rightarrow \infty}\left\|R_{n, p} U_{n, p}^{-1}\right\|_{1} \leq M .
$$

Now let $f \in A(\Gamma)$. The series $\sum_{n \in \mathbb{Z}} \widehat{f}(n) \pi^{+}(\alpha)^{n}$ converges in $A^{+} / J^{+}(E)$, and we have

$$
i\left(\sum_{n \in \mathbb{Z}} \widehat{f}(n) \pi^{+}(\alpha)^{n}\right)=\sum_{n \in \mathbb{Z}} \widehat{f}(n) \pi(\alpha)^{n}=\pi(f) .
$$

Hence $i$ is onto, and (1) holds. Notice that the arguments above show that the $A A^{+}$-constant of $E$ is the smallest constant $M$ for which there exists a sequence $\left(\psi_{n}\right)$, which converges $w^{*}$ to 1 , such that $\limsup _{n \rightarrow \infty}\left\|\psi_{n}\right\| \leq M$.

If (1) holds, let $p \geq 1$ and $\varepsilon>0$; denote again by $K$ the strong $A A^{+}$-constant of $E$. There exist $f \in A^{+}$and $g \in J(E)$ such that $1=\alpha^{n+p} f+g$, so that $\alpha^{-n}=\alpha^{p} f+g \alpha^{-n}$, with $\|f\|_{1} \leq K+\varepsilon$.

Let $S \in P M(E)$. We obtain

$$
|\widehat{S}(p)|=\left|\left\langle\alpha^{-p} f, S\right\rangle\right|=\left|\sum_{n=0}^{\infty} \widehat{f}(n) \widehat{S}(-p-n)\right| \leq(K+\varepsilon) \sup _{n \geq 0}|\widehat{S}(-n-p)| .
$$

Hence $\|S\|_{P M} \leq K \lim \sup _{n \rightarrow \infty}|\widehat{S}(-n)|$, and (3) holds.

The fact that (3) implies (1), and the assertion concerning the $A A^{+}$-constant of $E$, follow from the same duality argument as in [16, Theorem 2.1].

Strong $A A^{+}$-sets give a large class of sets of uniqueness. The simplest example is given by countable sets [16, Theorem 3.1] since pseudomeasures with countable support are almost periodic. Other examples are given by $H$-sets, i.e. sets $E$ 
such that there exists a strictly increasing sequence $\left(p_{n}\right)$ of integers such that $\bigcup_{n \geq 1} E^{p_{n}}$ is not dense in $\Gamma$ (the argument given in [16] shows that $H$-sets are strong $A A^{+}$-sets). It is immediate that $E_{1 / p}$ is an $H$-set if $p \geq 3$ is an integer, and this remains true for $E_{\zeta}$ if $1 / \zeta$ is a quadratic Pisot number. The sets $E_{\zeta}$, where $1 / \zeta$ is a Pisot number of degree $\geq 3$, belong to the more general class of $H^{(p)}$-sets of Pyateckiu-Shapiro [17, p. 59]. These sets are also strong $A A^{+}$-sets-this also follows from the proof of [16, Theorem 3.3].

Definition 2.11. Let $E \subset \Gamma$ be a closed set.

(1) $E$ is a Kronecker set if for every $f \in \mathcal{C}(E)$ with $|f(z)| \equiv 1$ on $E$, there exists a sequence $\left(p_{n}\right)$ of integers such that $\lim _{n \rightarrow \infty} z^{p_{n}}=f(z)$ uniformly on $E$.

(2) $E$ is a Dirichlet set if there exists a strictly increasing sequence $\left(p_{n}\right)$ of integers such that $\lim _{n \rightarrow \infty} z^{p_{n}}=1$ uniformly on $E$.

(3) $E$ is a Helson set if $A(E)=\mathcal{C}(E)$.

Any finite set is a Dirichlet set, and any finite independent set is a Kronecker set [17, appendix 5].

A Kronecker set is a Dirichlet set and a Helson set, and is of synthesis by a theorem of Varopoulos [30]. Clearly, Dirichlet sets are $H$-sets, and Dirichlet sets have strong $A A^{+}$-constant 1 [15, p. 98].

Helson sets are $A A^{+}$-sets by a theorem of Wik [31], so that the condition $A(E)=\mathcal{C}(E)$ ensures in fact that $A^{+}(E)=\mathcal{C}(E)$. In particular, $I^{+}(E)$ is $w^{*}$ sequentially dense in $A^{+}$, and this argument seems new (the weaker fact that $I(E)$ is $w^{*}$-sequentially dense in $A(\Gamma)$ is proved in [11, Theorem 4.5.2] by more complicated arguments). We refer to the classical books [11], [15], [17] for further information (sets of uniqueness are closed under closed countable unions, there are sets of uniqueness such that $J(E)$ is not sequentially $w^{*}$-dense in $A(\Gamma)$, etc. . ) and we conclude this section by the famous and surprising result of Körner and Kaufman.

THEOREM 2.12 [19], [22]. Every set of multiplicity contains a Helson set of multiplicity.

\section{Strong uniqueness properties of countable sets}

Definition 3.1. $H D^{0}(\Gamma)=\left\{S \in H D(\Gamma) \mid \lim _{n \rightarrow-\infty} \widehat{S}(n)=0\right\}$. If $E \subset \Gamma$ is closed, $H D^{0}(E)=H D^{0}(\Gamma) \cap H D(E)$.

We are interested here in geometric conditions on $E$ which ensure that every element $S$ of $H D^{0}(E)$ such that $(\widehat{S}(n))_{n \geq 0}$ satisfies some growth condition vanishes.

Of course, $P F(\Gamma) \subset H D^{0}(\Gamma)$. We now introduce examples of elements of $H D^{0}(\Gamma)$ related to inner functions. Let $E \subset \Gamma$ be closed, and let $\mu$ be a positive 
singular measure concentrated on $E$. Let

$$
F_{\mu}(z)=\exp \left(\frac{1}{2 \pi} \int_{0}^{2 \pi} \frac{e^{i t}+z}{e^{i t}-z} d \mu(t)\right)-\exp (-\|\mu\|) \quad(z \in \mathbb{C} \backslash E)
$$

where

$$
\|\mu\|=\frac{1}{2 \pi} \int_{0}^{2 \pi} d \mu(t)
$$

is the total variation of $\mu$. Then $F_{\mu} \in H D(\Gamma)$, the function

$$
z \rightarrow \frac{1}{F_{\mu}(z)+\exp (-\|\mu\|)} \quad(z \in D)
$$

is inner, and the function $z \rightarrow F_{\mu}(1 / z)+\exp (-\|\mu\|)(|z|<1)$, extended by continuity at 0 , is also inner. This shows that

$$
\sum_{n \leq 0}\left|\widehat{F}_{\mu}(n)\right|^{2}<\infty
$$

and so $F_{\mu} \in H D^{0}(\Gamma)$.

If $\mu=2 \pi \lambda \delta_{t_{0}}$ and $z_{0}=e^{i t_{0}}$, we have

$$
F_{\mu}(z)=\exp \left(\lambda \frac{z_{0}+z}{z_{0}-z}\right)-e^{-\lambda} .
$$

Routine computations using Cauchy estimates lead to the following observation (see [2], [9], [33]).

Proposition 3.2. For every $z_{0} \in \Gamma$, and every $\varepsilon>0$, there exists a nonzero $S_{\varepsilon} \in H D^{0}\left\{z_{0}\right\}$ such that

$$
\limsup _{n \rightarrow \infty} \frac{\log ^{+}\left|S_{\varepsilon}(n)\right|}{\sqrt{n}}<\varepsilon .
$$

Now let $E$ be an uncountable, closed subset of $\Gamma$. It is well known that $E$ contains a closed, perfect set $F$. If $F$ has positive Lebesgue measure, then $F$ is a set of multiplicity. If $F$ has zero Lebesgue measure, then $F$ is the support of a positive, singular, nonatomic measure $\mu$. Applying Cauchy's inequalities to $F_{\mu}$, and using standard estimates of Poisson integrals, we also obtain the following result $[34]$.

Proposition 3.3. For every uncountable closed set $E \subset \Gamma$ there exists a nonzero $S \in H D^{0}(E)$ such that

$$
\limsup _{n \rightarrow \infty} \frac{\log ^{+}|\widehat{S}(n)|}{\sqrt{n}}=0 .
$$

Now set

$$
\mathcal{E}=\left\{S \in H D^{0}(\Gamma)\left|\log ^{+}\right| \widehat{S}(n) \mid / \sqrt{n} \underset{n \rightarrow \infty}{\longrightarrow} 0\right\}
$$


(Using Cauchy's inequalities, it is easy to see that

$$
\left.\mathcal{E}=\left\{S \in H D^{0}(\Gamma)|(1-|z|) \log | S^{+}(z) \mid \underset{|z| \rightarrow 1^{-}}{\longrightarrow} 0\right\} .\right)
$$

We now wish to prove, by analytic methods, that the support of every nonzero element of $\mathcal{E}$ is a perfect set, a result implicitly contained in [34]. First, we need to discuss decompositions of elements of $H D(\Gamma)$ with disconnected support. If $S \in H D(\Gamma)$, then

$$
\frac{1}{2 i \pi} \int_{\Gamma(0, r)} \frac{S(\zeta)}{\zeta-z} d \zeta=0 \quad \text { for } r>\sup (1,|z|)
$$

where $\Gamma(a, r)$ is the circle $\{\zeta \in \mathbb{C}|| \zeta-a \mid=r\}$ oriented positively.

Now if $K$ and $L$ are disjoint closed subsets of the circle such that $\operatorname{Supp} S \subset$ $K \cup L$ it is easy, using some suitable contour integrals and the Cauchy formula, to construct $S_{K}, S_{L} \in H D(\Gamma)$ such that $S_{K}+S_{L}=S$ and $\operatorname{Supp} S_{K} \subset K$, $\operatorname{Supp} S_{L} \subset L$. This decomposition is unique, since $K$ and $L$ are disjoint.

If $K=\left\{z_{0}\right\}$, which means that there exists $\varrho>0$ such that $\operatorname{Supp} S \cap D\left(z_{0}, \varrho\right) \subset$ $\left\{z_{0}\right\}$, we have

$$
S_{K}(z)=-\frac{1}{2 i \pi} \int_{\Gamma\left(z_{0}, \varepsilon(z)\right)} \frac{S(\zeta)}{\zeta-z} d \zeta
$$

where $\varepsilon(z)$ is any element of $\left(0, \inf \left(\varrho,\left|z_{0}-z\right|\right)\right)$.

We have the following lemma (the author was not able to avoid the use of regular Beurling algebras to prove it).

Lemma 3.4. Let $S \in \mathcal{E}$, and let $K, L$ be disjoint closed subsets of $\Gamma$ such that Supp $S \subset K \cup L$. Then $S_{K} \in \mathcal{E}$ and $S_{L} \in \mathcal{E}$.

Proof. Routine elementary computations [33] that we omit show that there exists a submultiplicative weight $\omega: \mathbb{N} \rightarrow[1, \infty[$ such that

$$
\sup _{n \geq 0} \frac{|\widehat{S}(n)|}{\omega(n)}<\infty \quad \text { and } \quad \frac{\log \omega(n)}{\sqrt{n}} \underset{n \rightarrow \infty}{\longrightarrow} 0 .
$$

Let $\tau(n)=1(n \geq 0), \tau(n)=\omega(-n)(n<0)$ and consider

$$
A_{\tau}(\Gamma)=\left\{f \in \mathcal{C}(\Gamma)\left|\sum_{n \in \mathbb{Z}}\right| \widehat{f}(n) \mid \tau(n)<\infty\right\} .
$$

Then $A_{\tau}(\Gamma)$ is a regular Banach algebra with respect to the norm $\|f\|_{\tau}=$ $\sum_{n \in \mathbb{Z}}|\widehat{f}(n)| \tau(n)$ (see [18]), since

$$
\sum_{n \in \mathbb{Z}} \frac{\log \tau(n)}{1+n^{2}}<\infty .
$$

Let

$$
H D_{\tau}(\Gamma)=\left\{T \in H D(\Gamma)\left|\sup _{n \in \mathbb{Z}}\right| \widehat{T}(n) \mid / \tau(-n)<\infty\right\}
$$


Then we can identify isometrically $H D_{\tau}(\Gamma)$ with the dual space of $A_{\tau}(\Gamma)$ by the duality formula $\langle f, T\rangle=\sum_{n \in \mathbb{Z}} \widehat{f}(n) \widehat{T}(-n)$. Formula (2.4) and Proposition 2.6 remain valid (using synthesis with respect to $A_{\tau}(\Gamma)$ instead of synthesis for $A(\Gamma)$ ). For $g \in A_{\tau}(\Gamma)$ and $T \in H D_{\tau}(\Gamma)$ set $\langle f, g T\rangle=\langle f g, T\rangle\left(f \in A_{\tau}(\Gamma)\right)$. Then $g T \in$ $H D_{\tau}(\Gamma)$ and $\operatorname{Supp} g T \subset \operatorname{Supp} g \cap \operatorname{Supp} T$. Also $g T \in H D^{0}(\Gamma)$ if $T \in H D_{\tau}(\Gamma) \cap$ $H D^{0}(\Gamma)$. Since $A_{\tau}(\Gamma)$ is regular, there exist $g$ and $h$ such that Supp $g \cap L=\emptyset$, Supp $h \cap K=\emptyset, g+h \equiv 1$. Since $S \in H D^{0}(\Gamma) \cap H D_{\tau}(\Gamma)$ we have $g S \in \mathcal{E}$, $h S \in \mathcal{E}, \operatorname{Supp} g S \cap L=\emptyset$, Supp $g S \subset S$ so Supp $g S \subset K$.

Similarly Supp $h S \subset L$. Hence $S_{K}=g S, S_{L}=h S$, which proves the lemma.

Theorem 3.5. Let $S \in H D(\Gamma)$ such that

$$
\widehat{S}(n) \underset{n \rightarrow-\infty}{\longrightarrow} 0, \quad \frac{\log ^{+}|\widehat{S}(n)|}{\sqrt{n}} \underset{n \rightarrow \infty}{\longrightarrow} 0 .
$$

Then Supp $S$ is a perfect set.

Pro of. Assume that there exists $\varrho>0$ such that Supp $S \cap D\left(z_{0}, \varrho\right) \subset\left\{z_{0}\right\}$. We can assume without loss of generality that $z_{0}=1$ and $\varrho<1$. Denote by $\log \zeta$ the branch of the logarithm in $D(1, \varrho)$ which vanishes at 1 . For $z \in \mathbb{C}$ set

$$
F(z)=\frac{1}{2 i \pi} \int_{\Gamma(1, \varepsilon(z))} S(\zeta) \zeta^{z-1} d \zeta,
$$

where $\varepsilon(z) \in(0, \inf (\varrho,|z-1|))$.

Clearly, $F$ is an entire function of exponential type 0 , since the definition above does not depend on the choice of $\varepsilon(z)$. Let $T=S_{\{1\}}$. Then, since $S-T$ is holomorphic in $D(1, \varrho)$, we have

$$
F(z)=\frac{1}{2 i \pi} \int_{\Gamma(1, \varepsilon(z))} T(\zeta) \zeta^{z-1} d \zeta .
$$

By integrating $T(\zeta) \zeta^{z-1}$ over two suitable other circles of radii respectively smaller and larger than 1 centered at the origin, we deduce from Cauchy's theorem that $F(n)=-\widehat{T}(-n)(n \in \mathbb{Z})$. Hence

$$
F(n) \underset{n \rightarrow \infty}{\longrightarrow} 0, \quad \frac{\log ^{+}|F(n)|}{\sqrt{n}} \underset{n \rightarrow-\infty}{\longrightarrow} 0 .
$$

Since $\lim _{n \rightarrow \infty} F(n)=0$ and since $F$ is of exponential type 0 , it follows from Cartwright's theorem [4, Theorem 10.2.1] that $\lim _{t \rightarrow \infty} F(t)=0$. Now define $\sqrt{-i z}$, for $\operatorname{Im} z \geq 0$, by taking the argument of $-i z$ in $[-\pi / 2, \pi / 2]$ and set $G_{\varepsilon}(z)=e^{-\varepsilon \sqrt{-i z}}(\operatorname{Re} z \geq 0)$. Since $\sup _{n \in \mathbb{Z}}\left|F(n) G_{\varepsilon}(n)\right|<\infty$ it follows again from Cartwright's theorem that $F G_{\varepsilon}$ is bounded on the real line, and it follows from the Phragmén-Lindelöf principle [4, Theorem 1.4.1] that $F G_{\varepsilon}$ is bounded in the upper half-plane. 
Hence

$$
\limsup _{\substack{|z| \rightarrow \infty \\ \operatorname{Im} z \geq 0}} \frac{\log ^{+}|F(z)|}{\sqrt{|z|}}=0
$$

Using a similar argument for the lower half-plane we see that in fact

$$
\limsup _{|z| \rightarrow \infty} \frac{\log ^{+}|F(z)|}{\sqrt{|z|}}=0 \text {. }
$$

But another standard application of the Phragmén-Lindelöf principle $[4$, Theorem 3.1.5] shows then that $F \equiv 0$, since $\lim _{x \rightarrow \infty} F(x)=0$. Hence $\widehat{T}(n)=0$ $(n \in \mathbb{Z}), T=0$, and 1 is regular for $S$ since $T=S_{\{1\}}$.

This shows that $\operatorname{Supp} S$ cannot have isolated points, and the theorem is proved.

4. Strong uniqueness properties of the Cantor set. We now use a very different argument to establish the following result (first proved in [9] by using the recent results about closed ideals of $A^{+}$).

THEOREM 4.1. Let $S$ be a distribution on $\Gamma$. If $\operatorname{Supp} S \subset E_{1 / p}$, and if $\lim _{n \rightarrow-\infty} \widehat{S}(n)=0$, then $S=0$.

Pr o of. Let $\mathcal{A}^{\infty}(D)$ be the algebra of infinitely differentiable functions on the closed unit disc $\bar{D}$ which are analytic on $D$.

Routine computations show that

$$
\int_{0}^{2 \pi} \log \frac{2}{\operatorname{dist}\left(e^{i t}, E_{1 / p}\right)} d t<\infty
$$

and it follows then from the Taylor-Williams improvement [29] of a result of Carleson [6] that there exists a nonzero $f \in \mathcal{A}^{\infty}(D)$ vanishing exactly on $E_{1 / p}$. Fix $q \in \mathbb{Z}$ and set $f_{n}(z)=z^{-q} f\left(z^{p^{n}}\right)(n \geq 1, z \in \Gamma)$. Then $f_{n}$ is infinitely differentiable on $\Gamma$, and $f_{n}$ vanishes on $E_{1 / p}$. Since $E_{1 / p}$ is perfect, $f_{n}^{(k)}$ vanishes also on $E_{1 / p}$ for $k \geq 1$.

It follows then from a standard result about distributions that $\left\langle f_{n}, S\right\rangle=0$ $(n \geq 1)$. We obtain

$$
0=\left\langle f_{n}, S\right\rangle=\sum_{m=0}^{\infty} \widehat{S}\left(q-m p^{n}\right) \widehat{f}(m) \quad(n \geq 1) .
$$

Hence

$$
|\widehat{S}(q)| \leq \sum_{m=1}^{\infty}|\widehat{f}(m)|\left|\widehat{S}\left(q-m p^{n}\right)\right| \leq\|f\|_{1} \sup _{m \geq 1}\left|\widehat{S}\left(q-m p^{n}\right)\right|
$$

for each $n \geq 1$. Since $\widehat{S}(m) \rightarrow 0$ as $m \rightarrow-\infty, \widehat{S}(q)=0$ for every $q$, and $S=0$.

Theorem 4.1 holds for every set $E$ such that the closure of $\bigcup_{n \geq 1} E^{k_{n}}$ satisfies the Carleson condition for some strictly increasing sequence $\left(k_{n}\right)$ of positive 
integers. Clearly, such sets are $H$-sets, but we shall see in the next section that Theorem 4.1 is not true in general for $H$-sets. By using the geometric properties of $E_{1 / p}$ and some variant of the Beurling-Pollard method [15, p. 61] it is possible to obtain the following stronger result, that we mention without proof.

TheOREM $4.2[9]$. Let $S \in H D^{0}\left(E_{1 / p}\right)$. If

$$
\limsup _{n \rightarrow \infty} \frac{\log ^{+}|\widehat{S}(n)|}{n^{\alpha}}<\infty \quad \text { for some } \alpha<\frac{\log p-\log 2}{2 \log p-\log 2},
$$

then $S=0$.

In the other direction, by using singular measures concentrated on $E_{1 / p}$ as in Section 3 , it is possible to construct nonzero $S \in H D^{0}\left(E_{1 / p}\right)$ such that

$$
\limsup _{n \rightarrow \infty} \frac{\log ^{+}|\widehat{S}(n)|}{n^{\beta}}>0, \quad \text { where } \beta=\frac{2 \log p-\log 2}{4 \log p}
$$

(see [26]). The best constant for which Theorem 4.2 holds is not known.

5. Distributions on Dirichlet sets. We now prove that Theorem 4.1 does not extend in general to Dirichlet sets. The following lemma is well known.

Lemma 5.1. Let $E \subsetneq \Gamma$ be a closed set. Then $I(E)+A^{+}$is dense in $A(\Gamma)$.

Proof. Let $S \in P M(\Gamma)$ such that $S \perp\left(I(E)+A^{+}\right)$. Since $S \perp I(E), S \perp J(E)$ and so Supp $S \subsetneq \Gamma$. Since $S \perp A^{+}, \widehat{S}(n)=0(n \leq 0)$ and so $S(z)=0$ if $|z|>1$. By the principle of analytic continuation, $S=0$.

If $S \in P M(\Gamma)$ and $f \in A(\Gamma)$ we define $f S$ by the formula

$$
\langle g, f S\rangle=\langle f g, S\rangle \quad(g \in A(\Gamma)),
$$

so that $f S \in P M(\Gamma)$. Clearly, $\widehat{f S}(n)=\sum_{p \in \mathbb{Z}} \widehat{f}(p) \widehat{S}(n-p)(n \in \mathbb{Z})$, and $\operatorname{Supp} f S \subset \operatorname{Supp} f \cap \operatorname{Supp} S$. Also $\|f S\|_{P M} \leq\|f\|_{1}\|S\|_{P M}$, where $\|S\|_{P M}=$ $\sup |\widehat{S}(n)|(n \in \mathbb{Z})$, and $f S \in P F(E)$ if $S \in P F(E)$.

Definition 5.2. Let $\omega: \mathbb{N} \rightarrow[1, \infty[$. Let

$$
P M_{\omega}(\Gamma)=\left\{S \in H D(\Gamma)\left|\sup _{n \leq 0}\right| \widehat{S}(n)\left|<\infty, \sup _{n>0}\right| \widehat{S}(n) \mid / \omega(n)<\infty\right\} .
$$

For $S \in P M_{\omega}(\Gamma)$ set

$$
\|S\|_{\omega}=\max \left(\sup _{n \leq 0}|\widehat{S}(n)|, \sup _{n>0}|\widehat{S}(n)| / \omega(n)\right) .
$$

Clearly, $P M_{\omega}(\Gamma)$ is a Banach space if $\lim _{\sup _{n \rightarrow \infty}} \omega(n)^{1 / n}<1$, and $P M_{\omega}(\Gamma) \cap$ $H D^{0}(\Gamma)$ is a closed subspace of $P M_{\omega}(\Gamma)$. We omit the proof of the following elementary lemma [9].

Lemma 5.3. Let $S \in P F(\Gamma)$. If $\lim _{n \rightarrow \infty} \omega(n)=\infty$, then $\lim _{n \rightarrow \infty}\left\|\alpha^{n} S\right\|_{\omega}=$ 0 . 
Lemma 5.4. Suppose $\lim _{n \rightarrow \infty} \omega(n)=\infty$ and let $S \in P F(\Gamma)$. Then for every $\varepsilon>0$ and every $q \geq 1$ there exist $p \geq q$ and $f \in A(\Gamma)$ which satisfy the following two conditions:

1) $\|f S-S\|_{\omega}<\varepsilon$.

2) Supp $f \subset\left\{z \in \Gamma|| z^{p}-1 \mid \leq \varepsilon\right\}$.

Proof. We may assume $S \neq 0$. Let $E=\{z \in \Gamma|| z-1 \mid \geq \varepsilon\}$. It follows from Lemma 5.1 that there exists a sequence $\left(g_{n}\right)$ in $I(E)$ and a sequence $\left(h_{n}\right)$ in $A^{+}$ such that $\lim _{n \rightarrow \infty}\left\|\alpha^{-1}-g_{n}+h_{n}\right\|_{1}=0$. We have $\lim _{n \rightarrow \infty}\left\|1+\alpha h_{n}-\alpha g_{n}\right\|_{1}=0$, and so $\lim _{n \rightarrow \infty} \widehat{\alpha g_{n}}(0)=1$. Let $f=\left[\widehat{\alpha g_{n}}(0)\right]^{-1} \alpha g_{n}$ where $n$ is chosen so that

$$
\sum_{m<0}|\widehat{f}(m)|<\frac{\varepsilon}{2\|S\|_{P M}}
$$

We have $f \in I(E)$. For $p \geq 1$ define $f_{p}(z)=f\left(z^{p}\right)(z \in \Gamma)$. Clearly, Supp $f_{p} \subset$ $\left\{z \in \Gamma|| z^{p}-1 \mid \leq \varepsilon\right\}$. Also

$$
f_{p} S-S=\sum_{m<0} \widehat{f}(m) \alpha^{p m} S+\sum_{m>0} \widehat{f}(m) \alpha^{p m} S
$$

the series above being norm convergent in $P M(\Gamma)$. Since $\|T\|_{\omega} \leq\|T\|_{P M}(T \in$ $P M(\Gamma))$ we obtain

$$
\left\|f_{p} S-S\right\|_{\omega} \leq\left(\sum_{n<0}|\widehat{f}(n)|\right)\|S\|_{P M}+\|f\|_{1} \sup _{n \geq p}\left\|\alpha^{n} S\right\|_{\omega}
$$

and it follows from Lemma 5.3 that

$$
\limsup _{p \rightarrow \infty}\left\|f_{p} S-S\right\|_{\omega}<\varepsilon
$$

The lemma follows.

We set $D^{0}(\Gamma)=\left\{S \in D(\Gamma) \mid \lim _{n \rightarrow-\infty} \widehat{S}(n)=0\right\}$. We now show that Theorem 4.1 is not true in general for Dirichlet sets.

TheOREM 5.5. Let $E$ be a set of multiplicity, and let $\omega: \mathbb{N} \rightarrow[1, \infty[$ be such that $\lim _{n \rightarrow \infty} \omega(n)=\infty$. Then there exists a nonzero $S \in D^{0}(\Gamma)$ which satisfies the following conditions:

1) Supp $S \subset E$, and $\operatorname{Supp} S$ is a Dirichlet set.

2) $\sup _{n \geq 1}|\widehat{S}(n)| / \omega(n)<\infty$.

Proof. By setting $\omega_{1}(n)=\inf (\omega(n), n)(n \geq 1)$ we can assume without loss of generality that $\omega(n) \leq n$, so that $P M_{\omega}(\Gamma) \subset D(\Gamma)$. Let $S \in P F(E), S \neq 0$. It follows from Lemma 5.4 that we can define by induction a sequence $\left(f_{n}\right)_{n>1}$ in $A(\Gamma)$ and a strictly increasing sequence $\left(p_{n}\right)$ of positive integers which satisfy the following conditions:

(1) $\left\|S-f_{1} S\right\|_{\omega}<\|S\|_{\omega} / 4$.

(2) $\left\|f_{1} \ldots f_{n+1} S-f_{1} \ldots f_{n} S\right\|_{\omega}<\|S\|_{\omega} \cdot 2^{-n-2}(n \geq 1)$.

(3) $\operatorname{Supp} f_{n} \subset\left\{z \in \Gamma|| z^{p_{n}}-1 \mid \leq 1 / n\right\}$. 
It follows from (1) and (2) that there exists a nonzero $T \in P M_{\omega}(\Gamma)$ such that $\lim _{n \rightarrow \infty}\left\|f_{1} \ldots f_{n} S-T\right\|_{\omega}=0$. Set $V_{n}=\operatorname{Supp} f_{1} \ldots f_{n} S$ and let $\varphi$ be a smooth function on $\Gamma$ such that $\operatorname{Supp} \varphi \cap\left(\bigcap_{n \geq 1} V_{n}\right)=\emptyset$. There exists $n_{0} \geq 1$ such that $\operatorname{Supp} \varphi \cap V_{n}=\emptyset$ for $n \geq n_{0}$. Hence $\langle\varphi, T\rangle=\lim _{n \rightarrow \infty}\left\langle\varphi, f_{1} \ldots f_{n} S\right\rangle=0$. This shows that $\operatorname{Supp} T \subset \bigcap_{n>1} V_{n}$, and so $\operatorname{Supp} T$ is a Dirichlet set contained in $E$. Since $H D^{0}(\Gamma) \cap P M_{\omega}(\Gamma)$ is closed, $\lim _{n \rightarrow-\infty} \widehat{T}(n)=0$ and the theorem is proved.

Theorem 5.5, Lemma 5.3 and Lemma 5.4 remain true if we replace $P F(\Gamma)$ by $P M^{0}(\Gamma)=\left\{S \in P M(\Gamma) \mid \lim _{n \rightarrow-\infty} \widehat{S}(n)=0\right\}$. A refinement of the proof of Theorem 5.5 and Lemma 5.4 gives Kronecker sets instead of Dirichlet sets [9]. The nature of the construction has some analogies with Kaufman's construction of a Helson set of multiplicity ([17] and [11, p. 117]). Notice that if we apply Theorem 5.5 to $E_{\zeta}$, where $1 / \zeta$ is not a Pisot number, we obtain a Dirichlet set which satisfies the Carleson condition and is even a set of " $\mathcal{A}{ }^{\infty}$-interpolation" in the sense of [1] (see [9]).

\section{Negative powers of contractions}

DeFinition 6.1. Let $\omega: \mathbb{N} \rightarrow[1, \infty[$ be a submultiplicative weight such that

$$
\lim _{n \rightarrow \infty} \frac{\log \omega(n)}{\sqrt{n}}=0 .
$$

A closed set $E \subset \Gamma$ is $\omega$-rigid if $P M_{\omega}(E)=P M(E)$.

It follows from the considerations at the beginning of Section 3 that $P M_{\omega}(E)$ $\supsetneq P M(E)$ for every nonempty set $E$ if $\liminf _{n \rightarrow \infty}(\log \omega(n)) / \sqrt{n}>0$, and so the condition $\lim _{n \rightarrow \infty}(\log \omega(n)) / \sqrt{n}=0$ is not a real restriction.

It was proved in [9] that for every submultiplicative weight $\omega$ such that $\lim _{n \rightarrow \infty}(\log \omega(n)) / \sqrt{n}=0$ there exists a perfect Kronecker set $E_{\omega} \subset \Gamma$ which is $\omega$-rigid.

If $\omega$ is a submultiplicative weight, and $E$ a closed set, set $P M_{\omega}^{0}(E)=H D^{0}(\Gamma) \cap$ $P M_{\omega}(E)$.

The following characterization of $\omega$-rigid sets is given in [9].

THEOREM 6.2. Let $E \subsetneq \Gamma$ be a closed set and let $\omega$ be a submultiplicative weight such that $\lim _{n \rightarrow \infty} \omega(n)=\infty$ and $\lim _{n \rightarrow \infty}(\log \omega(n)) / \sqrt{n}=0$. Then the following conditions are equivalent:

1) E is w-rigid.

2) For every Banach space $X$ and every linear contraction $T$ on $X$ such that $\operatorname{Sp} T \subset E$ and $\sup _{n \geq 1}\left\|T^{-n}\right\| / \omega(n)<\infty$, we have $\sup _{n \geq 1}\left\|T^{-n}\right\|<\infty$.

3) $E$ is a strong $A A^{+}$-set, and $P M_{\omega}^{0}(E)=\{0\}$.

Moreover, if $E$ is $\omega$-rigid and if $T$ satisfies 2) then $\sup _{n \geq 1}\left\|T^{-n}\right\| \leq K$ where $K$ is the strong $A A^{+}$-constant of $E$. 
The class of sets $E$ satisfying $P M_{\omega}^{0}(E)=\{0\}$ is stable under closed countable unions [9], and the class of strong $A A^{+}$-sets is not. So it would certainly be possible, using the method of [16] for constructing sets $E$ for which $\left\|e^{-i n t}\right\|_{A^{+}(E)}$ grows fast as $n \rightarrow \infty$, to exhibit sets satisfying $P M_{\omega}^{0}(E)=\{0\}$ which are not $\omega$ rigid. We will not do it here (notice that countable, not necessarily closed, unions of compact sets of uniqueness are sets of uniqueness by a theorem of N. Bari; see [7] for extensions of this result).

Using Theorems $6.2,3.5$ and 4.2 , we obtain for example the following consequence of Theorem 6.2 (countable sets are sets of synthesis and $A A^{+}$-sets of constant 1 , and $E_{1 / p}$ is a set of synthesis [12] and an $A A^{+}$-set [15], since it is an $H$-set).

TheOREM 6.3. Let $T$ be a linear invertible contraction on a Banach space $X$.

1) [34] If $\operatorname{Sp} T$ is countable, and if $\lim _{n \rightarrow \infty}\left(\log \left\|T^{-n}\right\|\right) / \sqrt{n}=0$, then $T$ is an isometry.

2) [9] If $\operatorname{Sp} T \subset E_{1 / p}$, and if

$$
\log \left\|T^{-n}\right\|=O\left(n^{\alpha}\right) \quad \text { with } \quad \alpha<\frac{\log p-\log 2}{2 \log p-\log 2},
$$

then $\sup _{n \geq 1}\left\|T^{-n}\right\| \leq K_{p}$, where $K_{p}$ is the $A A^{+}$-constant of $E_{1 / p}$.

In the other direction, it follows from Theorem 6.2 that if $E$ is a set of multiplicity there exists, for any weight $\omega$ such that $\lim _{n \rightarrow \infty} \omega(n)=\infty$, a Banach space $X_{\omega}$ and an invertible contraction $T_{\omega}$ on $X_{\omega}$ such that $\lim _{n \rightarrow \infty}\left\|T_{\omega}^{-n}\right\|=\infty$, $\operatorname{Sp} T_{\omega} \subset E$ and $\sup _{n \geq 1}\left\|T_{\omega}^{-n}\right\| / \omega(n)<\infty$. Since some sets of multiplicity satisfy the Carleson condition

$$
\int_{-\pi}^{\pi} \log ^{+}\left(\frac{1}{\operatorname{dist}\left(e^{i t}, E\right)}\right) d t<\infty
$$

(for example $E_{\zeta}$ when $1 / \zeta$ is not a Pisot number) the following result, implicitly contained in [9], shows that the situation is very different when we restrict attention to the Hilbert space.

TheOREm 6.4. Let $T$ be an invertible contraction on a Hilbert space $H$. If $\left\|T^{-n}\right\|=O\left(n^{k}\right)$ for some $k \geq 0$, and if $\operatorname{Sp} T$ satisfies the Carleson condition, then $T$ is unitary.

Proof. Let $x, y \in H$. Let $F(z)=\left\langle(T-z)^{-1} x, y\right\rangle(z \notin \operatorname{Sp} T)$. Then $F \in D(\Gamma)$, and $\operatorname{Supp} F \subset \operatorname{Sp} T$. For $f \in \mathcal{C}^{\infty}(\Gamma)$ set $f(T)=\sum_{n \in \mathbb{Z}} \widehat{f}(n) T^{n}$. Since $\widehat{F}(n)=T^{-n}$ $(n \in \mathbb{Z})$ we obtain

$$
\langle f, F\rangle=\sum_{n \in \mathbb{Z}} \widehat{f}(n) \widehat{F}(-n)=\langle f(T) x, y\rangle \quad\left(f \in \mathcal{C}^{\infty}(\Gamma)\right) .
$$

It follows from [29] that there exists $f \in \mathcal{A}^{\infty}(D), f$ outer, such that $\left.f^{(n)}\right|_{\operatorname{Sp} T}=0$ for every $n \geq 1$ and such that $f$ vanishes exactly on $\operatorname{Sp} T$. Since $\operatorname{Supp} F \subset \operatorname{Sp} T$ we have $\langle f(T) x, y\rangle=\langle f, F\rangle=0$. Hence $f(T)=0$. 
Since $T$ is a contraction on $H$, it follows from the classical von Neumann inequality that there exists a norm decreasing homomorphism $\theta: \varphi \rightarrow \varphi(T)$ from the disc algebra $\mathcal{A}(D)$ into $\mathcal{L}(H)$ such that $\alpha(T)=T$, where we denote by $\alpha$ the position function $z \rightarrow z$ (see for example [24] for details). Clearly, the two definitions of $\varphi(T)$ agree for $\varphi \in \mathcal{A}^{\infty}(D)$, and so $f \in \operatorname{Ker} \theta$.

Since $f$ is outer and vanishes exactly on $\operatorname{Sp} T$ it follows from the BeurlingRudin characterization of closed ideals of $\mathcal{A}(D)$ [27] that $\mathcal{M} \subset \operatorname{Ker} \theta$ where $\mathcal{M}=\left\{\varphi \in \mathcal{A}(D)|\varphi|_{\mathrm{Sp} T}=0\right\}$. It follows from classical results [13] that the restriction map $r:\left.f \rightarrow f\right|_{\operatorname{Sp} T}$ from $\mathcal{A}(D)$ into $\mathcal{C}(\operatorname{Sp} T)$ is onto and that the map $\widetilde{r}: \mathcal{A}(D) / \mathcal{M} \rightarrow \mathcal{C}(\operatorname{Sp} T)$ satisfying $\widetilde{r} \circ \pi=r$ is an isometry (we denote by $\pi: \mathcal{A}(D) \rightarrow \mathcal{A}(D) / \mathcal{M}$ the canonical map). Hence $\left\|\pi(\alpha)^{-1}\right\|=1$. Let $\widetilde{\theta}: \mathcal{A}(D) / \mathcal{M} \rightarrow \mathcal{L}(H)$ be the map satisfying $\widetilde{\theta} \circ \pi=\theta$. Then $T^{-1}=\tilde{\theta}\left(\pi(\alpha)^{-1}\right)$ and so $\left\|T^{-1}\right\|=1$ and $T$ is unitary.

The proof of Theorem 6.4 does not extend to the case where $\operatorname{Sp} T$ is not a Carleson set (in this case no nonzero analytic function satisfying a Lipschitz condition can vanish on $\operatorname{Sp} T$, see [6]), but of course Theorem 6.4 remains valid for some non-Carleson sets (consider a suitable convergent sequence). The subject clearly deserves further investigation.

7. Closed ideals of $A^{+}$. The investigations about strong forms of uniqueness and " $\omega$-rigidity" were originally motivated by problems concerning closed ideals of $A^{+}$(or, equivalently, closed invariant subspaces for the shift on $\ell^{1}\left(\mathbb{Z}^{+}\right)$). Let $I \neq\{0\}$ be a closed ideal of $A^{+}$, let $I^{A}$ be the set of elements of $A^{+}$which belong to the closed ideal generated by $I$ in $A(\Gamma)$, and let $S_{I}$ be the G.C.D. of the inner factors of all nonzero elements of $I$. Bennett and Gilbert [3] conjectured that we always have $I=I^{A} \cap S_{I} H^{\infty}$ (the conjecture was also quoted by Kahane [14]), and a similar conjecture has been verified for $\mathcal{A}^{\infty}(D)$ (Taylor-Williams [29]), $\mathcal{A}^{p}(D)$ (Korenblyum [21]), $\Lambda_{\alpha}(D)$, the algebra of analytic functions on $D$ satisfying a Lipschitz condition (Matheson [23]) and of course for the disc algebra $\mathcal{A}(D)$ (Beurling-Rudin, [27]). We refer to [9], [10] for a general discussion of the Bennett-Gilbert conjecture; for example, Theorem 3.5 can be used to show that the conjecture holds when $h(I)$ is countable, where $h(I)=\{z \in \bar{D} \mid f(z)=0$ $(f \in I)\}$, which was proved by Bennett-Gilbert by using transfinite induction [3] (transfinite induction was discovered and used by Cantor [5] to establish the fact that reducible countable sets are sets of uniqueness); also it is possible to deduce from Theorem 4.1 the fact that the Bennett-Gilbert conjecture holds when $h(I) \cap \Gamma \subset E_{1 / p}$ [10]. To conclude the paper, we will indicate how Theorem 5.5 can be used to disprove the conjecture. Let $\zeta \in(0,1 / 2)$ such that $1 / \zeta$ is not a Pisot number. Then $E_{\zeta}$ is a Carleson set.

It follows from Theorem 5.5 that there exists a Dirichlet set $F \subset E_{\zeta}$ such that $D^{0}(F) \neq\{0\}$. Let $J_{0}^{+}(F)$ be the closed ideal of $A^{+}$generated by the functions $f \in \mathcal{A}^{\infty}(D)$ vanishing on $F$ with all their derivatives. It follows from [29] that $J_{0}^{+}(F)$ contains an outer function vanishing exactly on $F$. Since smooth functions 
satisfy synthesis, $J(F)$ is the closed ideal of $A(\Gamma)$ generated by $J_{0}^{+}(F)$. So for $I=J_{0}^{+}(F)$ we obtain

$$
I^{A}=J^{+}(F), \quad S_{I}=1, \quad I^{A} \cap S_{I} H^{\infty}=J^{+}(F) .
$$

But $D^{0}(F) \neq\{0\}$, and $J^{+}(F)$ is $w^{*}$-sequentially dense in $A^{+}$, since Dirichlet sets are strong $A A^{+}$-sets. Hence $J_{0}^{+}(F) \subsetneq J^{+}(F)$, which disproves the conjecture.

\section{References}

[1] H. Alexander, B. A. Taylor and D. L. Williams, The interpolating sets for $A^{\infty}$, J. Math. Anal. Appl. 36 (1971), 556-566.

[2] A. Atzmon, Operators which are annihilated by analytic functions and invariant subspaces, Acta Math. 144 (1980), 27-63.

[3] C. Bennett and J. E. Gilbert, Homogeneous algebras on the circle: I. -Ideals of analytic functions, Ann. Inst. Fourier (Grenoble) 22 (3) (1972), 1-19.

[4] R. P. Boas, Entire Functions, Academic Press, New York, 1954.

[5] G. Cantor, Über die Ausdehnung eines Satzes aus der Theorie der trigonometrischen Reihen, Math. Ann. 5 (1872), 123-132.

[6] L. Carleson, Sets of uniqueness of functions regular in the unit circle, Acta Math. 87 (1952), 325-345.

[7] C. Carlet et G. Debs, Un résultat sur les ensembles d'unicité du tore, Publ. Math. Univ. Pierre et Marie Curie, no. 78, Sém. Init. Analyse 24 (1984-85), 191-193.

[8] I. Domar, On the analytic transform of bounded linear functionals on certain Banach algebras, Studia Math. 53 (1975), 203-224.

[9] J. Esterle, Distributions on Kronecker sets, strong forms of uniqueness and closed ideals of $A^{+}$, J. Reine Angew. Math., to appear.

[10] J. Esterle, E. Strouse and F. Zouakia, Closed ideals of $A^{+}$and the Cantor set, ibid., to appear.

[11] C. C. Graham and O. C. McGehee, Essays in Commutative Harmonic Analysis, Springer, Berlin, 1979.

[12] C. S. Herz, Spectral synthesis for the Cantor set, Proc. Nat. Acad. Sci. U.S.A. 42 (1956), $42-43$.

[13] K. Hoffman, Banach Spaces of Analytic Functions, Prentice-Hall, Englewood Cliffs, N.J., 1962.

[14] J.-P. Kahane, Idéaux fermés dans certaines algèbres de fonctions analytiques, in: Lecture Notes in Math. 336, Springer, 1973, 5-14.

[15] - Séries de Fourier Absolument Convergentes, Ergeb. Math. Grenzgeb. 50, Springer, Berlin, 1970.

[16] J.-P. Kahane et Y. Katznelson, Sur les algèbres de restrictions des séries de Taylor absolument convergentes à un fermé du cercle, J. Analyse Math. 23 (1970), 185-197.

[17] J.-P. Kahane et R. Salem, Ensembles Parfaits et Séries Trigonométriques, Hermann, Paris, 1963.

[18] Y. Katznelson, An Introduction to Harmonic Analysis, Wiley, New York, 1968.

[19] R. Kaufman, $M$-sets and distributions, Astérisque 5 (1973), 225-230.

[20] A. Kechris and A. Louveau, Descriptive Set Theory and the Structure of Sets of Uniqueness, London Math. Soc. Lecture Notes Ser. 128, Cambridge University Press, 1986.

[21] B. Korenblyum, Closed ideals in the ring $A^{n}$, Functional Anal. Appl. 6 (1972), 203-214.

[22] T. Körner, A pseudofunction on a Helson set. I, II, Astérisque 5 (1973), 3-224 and 231-239. 
[23] A. L. Matheson, Closed ideals in rings of analytic functions satisfying a Lipschitz condition, in: Lecture Notes in Math. 604, Springer, 1976, 67-72.

[24] B. Sz.- Nagy et C. Foiaş, Analyse Harmonique des Opérateurs de l'Espace de Hilbert, Akadémiai Kiadó, Budapest, 1967.

[25] I. I. Pyateckǐ̌-Shapiro, Supplement to the work "On the problem of uniqueness of expansion of a function in a trigonometric series", Moskov. Gos. Univ. Uchenye Zapiski 165 Mat. 7 (1954), 79-97 (in Russian).

[26] M. Rajoelina et M. Zarrabi, Sur les propriétés d'unicité forte de l'ensemble de Cantor, preprint.

[27] W. Rudin, The closed ideals in an algebra of analytic functions, Canad. J. Math. 9 (1957), 426-434.

[28] R. Salem et A. Zyg mund, Sur les ensembles parfaits dissymétriques à rapport constant, C. R. Acad. Sci. Paris Sér. A 240 (1955), 2281-2283.

[29] B. A. Taylor and D. L. Williams, Ideals in rings of analytic functions with smooth boundary values, Canad. J. Math. 22 (1970), 1266-1283.

[30] N. Varopoulos, Sur les ensembles parfaits et les séries trigonométriques, C. R. Acad. Sci. Paris Sér. A 260 (1965), 3831-3834.

[31] I. Wik, On linear independence in closed sets, Ark. Mat. 4 (1960), 209-218.

[32] W. H. Young, A note on trigonometric series, Mess. for Math. 38 (1909), 44-48.

[33] M. Zarrabi, Synthèse spectrale dans certaines algèbres de Beurling sur le cercle unité, Bull. Soc. Math. France (2) 118 (1990), 241-249.

[34] - Contractions à spectre dénombrable et propriétés d'unicité des fermés dénombrables du cercle, Ann. Inst. Fourier (Grenoble) 43 (1993), 251-263.

[35] F. Zouakia, Idéaux fermés de $A^{+}$et $L^{1}\left(\mathbb{R}^{+}\right)$, et propriétés asymptotiques des contractions et semi-groupes contractants, Thèse de Doctorat d'Etat, Bordeaux, 1990. 